\section{Spectral-domain OCT in anti-VEGF treatment of myopic choroidal neovascularization}

\begin{abstract}
Purpose To evaluate changes in macular morphology due to myopic choroidal neovascularization (CNV), using spectraldomain optical coherence tomography (SD-OCT).

Methods In all, 22 eyes with recent-onset untreated CNV underwent 1 intravitreal injection of anti-vascular endothelial growth factor (anti-VEGF), followed by a pro-re-nata regimen. SD-OCT was performed at baseline (before first administration of anti-VEGF treatment) and month 1, and 2; macular morphologic changes and outer retina characteristics (SD-OCT findings) associated with CNV activity were evaluated. Sensitivity and specificity were calculated for SD-OCT findings using fluorescein angiography (FA) as standard reference.
\end{abstract}

Results Mean central retinal thickness (CRT) showed no significant reduction from baseline ( $284 \pm 98 \mu \mathrm{m})$ to month

$1(257 \pm 74 \mu \mathrm{m})$ and month $2(263 \pm 72 \mu \mathrm{m})$.

Department of

Ophthalmology, Vita-Salute University, San Raffaele Scientific Institute, Milan, Italy

Correspondence:

U Introini, Department of Ophthalmology, Vita-Salute University, San Raffaele Scientific Institute, Via Olgettina 60, Milan 20132, Italy

Tel: + 393355606902 ;

Fax: +390226433643 .

E-mail: introini.ugo@hsr.it

Received: 4 January 2012 Accepted in revised form: 21 March 2012

Published online: 27 April 2012
A hyper-reflective lesion with fuzzy borders (fuzzy area), and 'absent or altered' IS/OS junction were the only SD-OCT findings associated with $\mathrm{CNV}$ activity $(P<\mathbf{0 . 0 0 0 1})$. Both these SD-OCT findings showed good sensitivity and specificity $(95.1$ and $\mathbf{9 6 . 0 \%}$ (95\% CI: $0.87-0.89$ ), respectively, for the fuzzy area; 87.9 and $66.7 \%$ (95\% CI: $0.65-0.87$ ), respectively, for 'absent or altered' IS/OS junction) when compared with FA leakage (standard reference).

Conclusions Outer retina characteristics (ie, hyper-reflective lesion with fuzzy borders, and 'absent or altered' IS/OS junction) appear more meaningful than CRT in the evaluation of myopic CNV activity. These SD-OCT findings show overall good sensitivity and specificity when compared with FA leakage (standard reference),
U Introini, G Casalino, G Querques, AT Gimeno, F Scotti and F Bandello

and could be considered as an alternative diagnostic tool to FA for myopic CNV monitoring.

Eye (2012) 26, 976-982; doi:10.1038/eye.2012.75; published online 27 April 2012

Keywords: spectral-domain OCT; pathologic myopia; choroidal neovascularization; anti-VEGF

\section{Introduction}

Pathologic myopia (PM) is one of the main causes of legal blindness in people under 50 years of age, ${ }^{1}$ due to the development of choroidal neovascularization (CNV) in the macula. The natural history of myopic $\mathrm{CNV}$ leads to irreversible impairment of visual function. ${ }^{2,3}$ How to best treat myopic CNV is still a matter of debate. Photodynamic therapy, which was widely used until a few years ago for subfoveal myopic CNV treatment, has failed to reveal statistically significant benefits at 2-year follow-up. ${ }^{4}$ Intravitreal anti-vascular endothelial growth factor (anti-VEGF) treatment has shown promising results, and is currently considered the first-line therapy for subfoveal and juxtafoveal myopic $\mathrm{CNV}^{5}$ although no multicenter trials have yet been published. The gold standard for myopic $\mathrm{CNV}$ diagnosis and monitoring is fluorescein angiography (FA). Optical coherence tomography (OCT) is a rapid, non-invasive imaging technique capable of generating optical cross-sections of the macula. The most widely used OCT devices, based on TD (time-domain) technology, have become fundamental instruments in the management of CNV complicating age-related macular degeneration (AMD), and this method is considered the gold standard when deciding 
re-treatment. ${ }^{6}$ Because of its low-level resolution, as well as the less exudative behaviour of these new vessels compared with those associated with neovascular AMD, little information regarding PM CNVs can be obtained from TD-OCT. Recently, the introduction of newgeneration high-resolution spectral-domain OCT devices (SD-OCT) into clinical practice has permitted precise evaluation of retinal morphology, and detailed visualization of retinal layers and pathological findings in the macula.

In this study, we used SD-OCT to evaluate macular morphological changes associated with active myopic $\mathrm{CNV}$, and determined their clinical significance in terms of sensitivity and specificity using FA as standard reference.

\section{Materials and methods}

We conducted a prospective non-randomized noncomparative monocentric interventional study. Patients presenting with myopic CNV between January 2009 and July 2010 at the Department of Ophthalmology of the Vita-Salute San Raffaele University in Milan, Italy were included. The research adhered to the tenets of the Declaration of Helsinki. Written informed consent was obtained for each patient after the potential risks and benefits of the intravitreal injections had been explained in detail. All applicable institutional and governmental regulations concerning the ethical use of human volunteers were followed during this study.

Selection inclusion criteria were: PM $(>-6 D)$ complicated by recent-onset active subfoveal or juxtafoveal CNV, as diagnosed by FA, and baseline best-corrected visual acuity (BCVA) of at least 20/200 determined with standard ETDRS (early treatment diabetic retinopathy study) charts. Exclusion criteria were: the presence of other retinal diseases or evidence of any condition other than PM associated with CNV, previous $\mathrm{CNV}$ treatment, intraocular surgery within the last 3 months, glaucoma not controlled by treatment, pregnancy, and other systemic conditions that might influence the treatment prognosis, that is, uncontrolled systemic hypertension and history of thromboembolic or ischaemic cardiovascular disease.

At baseline (before first administration of anti-VEGF treatment), and during all monthly visits, each patient underwent complete ophthalmological examination, including slit-lamp fundus biomicroscopy with dilated pupils, BCVA determined with standard ETDRS charts, and intraocular pressure with applanation tonometry. Fluorescein angiography (Retinal Camera TRC-50DX/ DX, Type IA, Topcon, Tokyo, Japan) was performed at baseline, month 1 and month 2 . FA images were captured after intravenous injection of $3 \mathrm{ml}$ of sodium fluorescein
$20 \%$, and were analysed by two independent retinal specialists (UI and ATG), who determined the presence of CNV activity. In the presence of disagreement concerning dye leakage, the opinion of a third retinal specialist $(\mathrm{FB})$ was taken as final.

SD-OCT (Spectral OCT/SLO, Opko/OTI, Miami, FL, USA) was also performed at baseline, month 1 , and month 2. SD-OCT scans were acquired using a $360^{\circ}$ radial line pattern, centred onto the fovea and passing through the CNV. On SD-OCT we recorded: central retinal thickness (CRT), outer retinal characteristics, including the presence of a hyper-reflective lesion above the retinal pigment epithelium (RPE), the presence of sub-retinal and intra-retinal fluid, and the status of the outer retinal lines, corresponding to the photoreceptor inner/outer segment (IS/OS) junction (also known as the ellipsoid portion of IS). ${ }^{7}$ CRT measurement was performed using the caliper provided by SD-OCT Opko/OTI image analysis software. The outer retinal lines were graded as 'present', or 'absent or altered' by two independent retinal specialists (UI and ATG), who were masked to FA findings. In the presence of disagreement regarding SD-OCT features, the opinion of a third retinal specialist (FB) was taken as final.

Following baseline evaluation, treatment consisted of a single intravitreal injection of anti-VEGF, either ranibizumab (Lucentis, Novartis, Basel, Switzerland) $0.5 \mathrm{mg}$, or bevacizumab (Avastin, Genentech, San Francisco, CA, USA) $1.25 \mathrm{mg}$, followed by a PRN (pro-re-nata) regimen. Both medications are currently 'off label' in the treatment of myopic CNV; because of the cost difference (ranibizumab costs approximately $\$ 2000$ per dose, whereas bevacizumab costs approximately $\$ 50),{ }^{8}$ treatment assignment was based on patient choice.

The injection procedure was performed in the operating room under sterile conditions with topical anaesthesia. Bevacizumab was provided by the local hospital pharmacy in a prefilled syringe. Patients used topical antibiotic drops for $72 \mathrm{~h}$ after injection.

After the first injection, re-treatment decision was based on the persistence of CNV leakage on FA, with or without BCVA decrease, or metamorphopsia.

The main outcome measures were the identification, on SD-OCT, of macular morphological changes associated with active myopic CNV, before and after antiVEGF treatment, and their clinical significance in terms of sensitivity and specificity, using FA as a standard reference. Treatment outcome measures included changes in mean BCVA expressed as a logarithm of the minimum angle of resolution ( $\log M A R)$, and changes in mean CRT as evaluated by SD-OCT.

Statistical calculations were performed using Statistical Package for Social Sciences (version 17.0, SPSS Inc., Chicago, IL, USA). Discrepancies between graders 
were judged using the Kappa measure of agreement. Qualitative data were compared using the Pearson chi-squared test. FA was considered as the standard reference. Sensitivity and specificity of SD-OCT (outer retina characteristics associated with $\mathrm{CNV}$ activity) were calculated. Binomial distribution was used to estimate 95\% confidence intervals. The Student's $t$-test was used to assess differences between paired means (BCVA LogMAR and CMT changes). The chosen level of statistical significance was $P<0.05$.

\section{Results}

A total of 22 eyes in 22 consecutive patients ( 9 men, 13 women; mean age $55 \pm 12$ years, range 33-71 years) were included in the analysis. The mean spherical equivalent refraction error was $-12.65 \pm 3.18$ dioptres. In all, 8 patients presented juxtafoveal $\mathrm{CNV}$, whereas 14 presented subfoveal CNV. At baseline, 9 eyes were treated with ranibizumab (Lucentis) $0.5 \mathrm{mg}$ and 13 eyes with bevacizumab (Avastin) $1.25 \mathrm{mg}$. There was no statistically significant difference in terms of age ( $57 \pm 10$ vs $55 \pm 13$ years, ranibizumab and bevacizumab, respectively; $P=0.9)$ and baseline BCVA $(0.41 \pm 0.28$ LogMAR vs $0.50 \pm 0.41$ LogMAR, ranibizumab and bevacizumab respectively; $P=0.8$ ) between the two treatment groups.

Overall, a total of 66 FA and SD-OCT examinations were performed from baseline to month 2 .

At baseline, all 22 eyes showed CNV leakage on FA. One out of twenty-two eyes $(4.5 \%)$, and three out of twenty-two eyes (13.6\%), still showed presence of FA leakage from the CNV at month 1 and 2, respectively. Grader agreement for the evaluation of CNV leakage on FA was: $K=0.92$. On the basis of these FA findings, 4 patients ( $3 / 4$ treated with bevacizumab, and $1 / 4$ treated with ranibizumab) needed more than one injection up to month 2 . The mean number of intravitreal injections during the study period was 1.18 (range 1-2). One eye received one additional injection at month 1 , and three eyes received one additional injection at month 2 .

At baseline, mean LogMAR BCVA was $0.46 \pm 0.28$; it significantly improved to $0.28 \pm 0.27(P=0.0001)$ and to $0.29 \pm 0.28(P=0.0001)$, at month 1 and 2 , respectively (Table 1). There was no statistical difference regarding BCVA at month 2 between the two treatment groups $(0.30 \pm 0.28$ LogMAR for bevacizumab, and $0.25 \pm 0.28$ LogMAR for ranibizumab; $P=0.64$ ). At baseline, mean CRT was $284 \pm 98 \mu \mathrm{m}$; it did not change significantly at both month 1 and $2(257 \pm 74 \mu \mathrm{m}, P=0.06$, and $263 \pm 72 \mu \mathrm{m}, P=0.09$, respectively) (Table 1 ). No ocular or systemic side effects were observed during the study period.
Table 1 Mean best-corrected visual acuity (BCVA) and mean SD-OCT central retinal thickness (CRT) changes during the study period

\begin{tabular}{lccc}
\hline & Baseline & Month 1 & Month 2 \\
\hline $\begin{array}{l}\text { Mean BCVA } \\
\text { LogMAR }) \pm \text { SD }\end{array}$ & $0.46( \pm 0.28)$ & $0.28( \pm 0.27)$ & $0.29( \pm 0.28)$ \\
& & $P=0.0001$ & $P=0.0001$ \\
$\begin{array}{l}\text { Mean CRT } \\
(\mu \mathrm{m}) \pm \text { SD }\end{array}$ & $284( \pm 98)$ & $257( \pm 74)$ & $263( \pm 72)$ \\
& & $P=0.06$ & $P=0.09$ \\
\hline
\end{tabular}

A $P$-value of $<0.05$ was considered statistically significant.

At baseline, SD-OCT scans passing through the CNV showed a hyper-reflective lesion with fuzzy borders (fuzzy area) and a more highly reflective core above the $\mathrm{RPE}$, associated with thickening of the overlying retina in 21 out of 22 eyes $(95.5 \%)$ (Figure 1a). In correspondence with the myopic $\mathrm{CNV}$, the IS/OS junction was 'absent or altered' in 19 out of 22 eyes (86.4\%) (Figure 2a). A slight amount of sub-retinal fluid and a few small intra-retinal cystic spaces were detected in 4 out of 22 eyes (18.2\%).

At month 1, the fuzzy area was not detectable in 20 out of 22 eyes (90.9\%) (Figure 1b), whereas it was still visible in only 2 eyes $(9.1 \%$ ) (2 out of 21 eyes showing this feature at baseline). The only eye showing CNV leakage on FA at month 1 also showed the fuzzy area. In correspondence with the myopic CNV, the IS/OS junction was 'absent or altered' in 6 out of 22 eyes (27.2\%), and was 'present' in 16 out of 22 eyes (72.7\%); all these latter eyes did not show CNV leakage on FA. In 17 eyes $(77.3 \%)$, in correspondence with the myopic CNV, the RPE appeared thickened (Figure 2b). A slight amount of sub-retinal fluid was still present in 1 out of 22 eyes $(4.5 \%)$ and few small intra-retinal cystic spaces were detected in another out of 22 eyes (4.5\%); these eyes did not show CNV leakage on FA.

At month 2, the fuzzy area was visible in 2 out of 22 eyes $(13.6 \%)$ (two different eyes from those that showed the fuzzy area at month 1). The 2 eyes showing the fuzzy area also showed CNV leakage on FA. In correspondence with the myopic CNV, the IS/OS junction was 'absent or altered' in 8 out of 22 eyes $(36.4 \%)$ and was 'present' in 14 out of 22 eyes $(63.6 \%) ; 13$ out of these 14 eyes did not show CNV leakage on FA, whereas 1 out of these 14 eyes showed CNV leakage on FA. In 18 eyes (81.8\%), in correspondence with the myopic CNV, the RPE appeared thickened. The same 2 eyes that showed a slight amount of sub-retinal fluid and a few small intra-retinal cystic spaces at month 1 continued to show the same at month 2; these eyes did not show CNV leakage on FA.

Grader agreement for evaluation of macular morphologic changes on SD-OCT was $K=0.94$. 

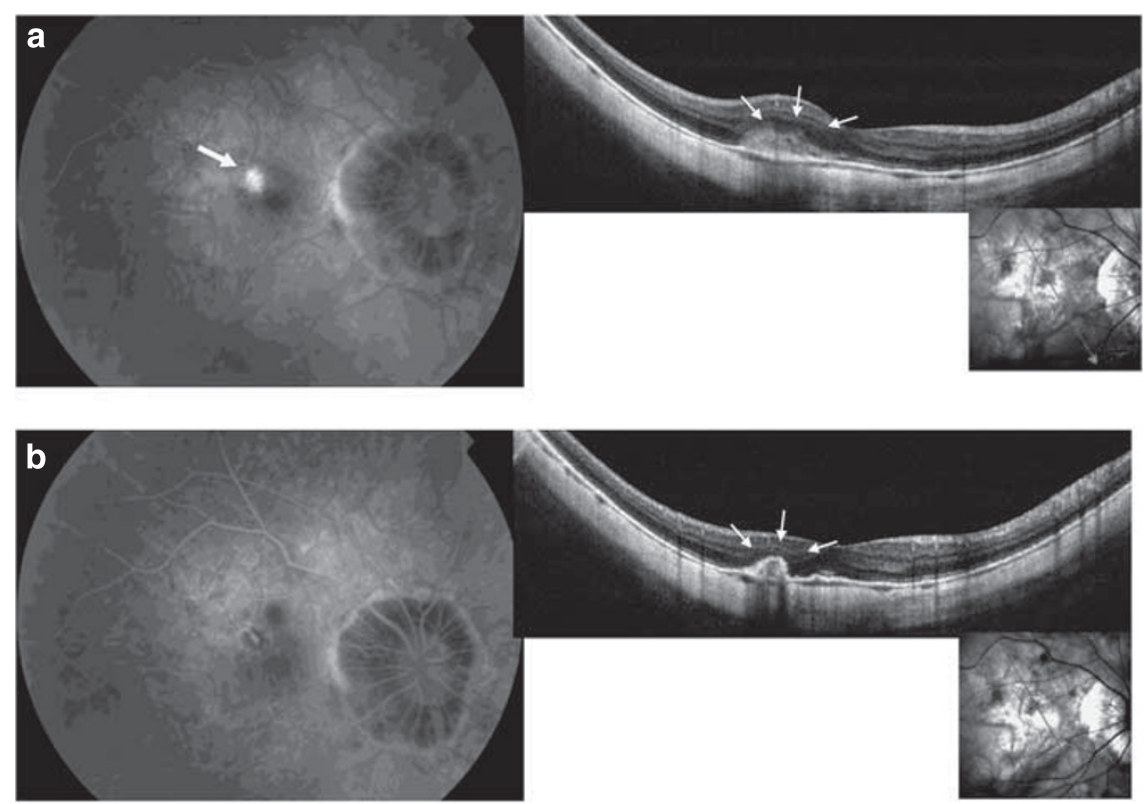

Figure 1 (a) An active leaking CNV is visible at baseline on FA (top left, white arrow). Baseline SD-OCT scan shows the hyperreflective area with fuzzy borders above the RPE (fuzzy area) corresponding to the CNV (top right, small arrows). (b) At month 1, no CNV leakage on FA is detectable (bottom left). SD-OCT scan shows the disappearance of the fuzzy area and a marked thickening of the hyper-reflective line corresponding to the RPE (bottom right, small arrows).
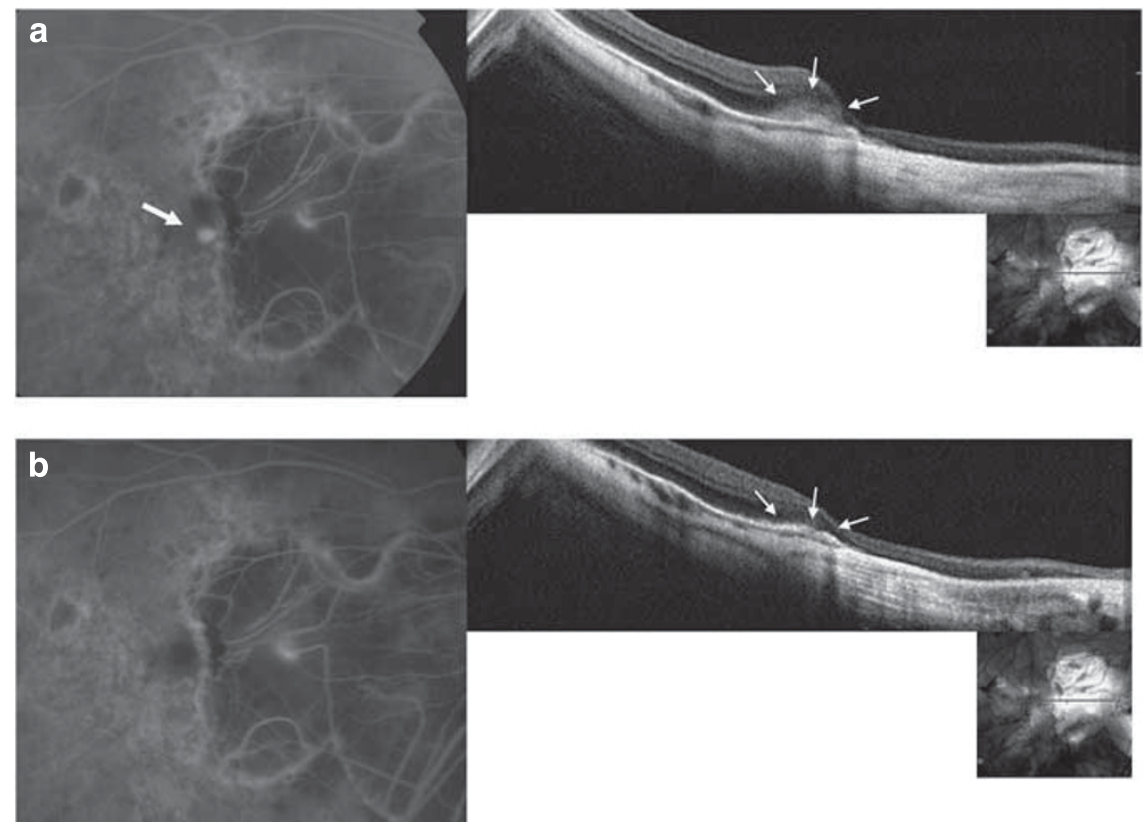

Figure 2 (a) A recent-onset $\mathrm{CNV}$ at the edge of the myopic peripapillary crescent is visible at baseline FA (top left, white arrow). Baseline SDOCT scan shows the fuzzy area (top right, small arrows) and the absence of the IS/OS junction. (b) At 1 month after antiVEGF treatment, no dye leakage on FA is detectable (bottom left). SD-OCT scan shows the disappearance of the fuzzy area and a slight thickening of the RPE (bottom right, small arrows).

The presence of sub-retinal and intra-retinal fluid was not associated with $\mathrm{CNV}$ activity $(P=0.07)$, whereas the fuzzy area, and 'absent or altered' IS/OS junction were the only outer retinal characteristics (SD-OCT findings) associated with CNV activity $(P<0.0001)$, in a total of 66 analysed examinations performed from baseline to month 2. Both these SD-OCT findings showed good sensitivity and specificity (95.1 and 96.0\% (95\% CI: 
0.87-0.89), respectively, for fuzzy area; 87.9 and $66.7 \%$ (95\% CI: 0.65-0.87), respectively, for 'absent or altered' IS/OS junction) when compared with FA leakage (standard reference).

\section{Discussion}

Randomized clinical trials have demonstrated the safety, efficacy, and superiority, compared with other treatment methods, of intravitreal anti-VEGFs for CNV due to AMD. 9,10 The Prospective OCT Imaging of Patients with Neovascular AMD Treated with Intra-Ocular Lucentis study $^{11}$ and the multicenter open label trial SUSTAIN, ${ }^{6}$ which used OCT-measured CRT and visual acuity as guiding criteria for re-treatment with a ranibizumab flexible dosing regimen, showed outcomes comparable overall to those of a monthly fixed regimen. Before the advent of OCT, FA was the only major diagnostic technique able to detect CNV activity. Simplicity, noninvasiveness and the absence of side effects are the main advantages of OCT compared with FA, and the reasons for its rapid success and development in the management of AMD patients (especially those undergoing anti-VEGF treatment needing close monitoring).

Small pilot studies have suggested the short-term benefits of anti-VEGFs in the treatment of PM-related CNV. ${ }^{12-17}$ As a consequence, there is a growing interest in the use of OCT in the management of CNV complicating PM. However, in PM CNV, OCT does not seem to provide information as meaningful as in AMD CNV. In fact, in myopic eyes, the retina and choroid are thin. Moreover, myopic CNV has minimal leakage, with scarce or no intra-retinal/sub-retinal fluid. Therefore, in myopic eyes OCT macular thickness evaluation during treatment appears irrelevant. ${ }^{18}$ In this study, we tested whether SD-OCT might permit the detection of macular morphological changes and outer retinal characteristics (SD-OCT findings) associated with CNV activity, and determined the clinical significance in terms of sensitivity and specificity (using FA as a standard reference). With this aim, we analysed the changes at the CNV site before and after administration of anti-VEGF treatment. Overall, the 22 included patients underwent 66 FA and SD-OCT examinations from baseline to month 2 . Treatment decision (PRN regimen) was based on the presence/ persistence of CNV leakage on FA. This allowed the identification of a hyper-reflective lesion with fuzzy borders and a more highly reflective core above the RPE, and 'absent or altered' IS/OS junction, as the outer retinal characteristics (SD-OCT findings) associated with CNV activity. These SD-OCT findings showed overall good sensitivity and specificity when compared with FA leakage (standard reference), and may be considered as valuable guiding criteria for the treatment of PM CNV with an anti-VEGF flexible dosing regimen.

In a prospective case series of 32 eyes affected by PM $\mathrm{CNV}$ treated with intravitreal injections of Ranibizumab, Lalloum et al ${ }^{17}$ reported significant macular thickness reduction on SD-OCT from baseline to final examination. Interestingly, in our study CRT did not significantly change after anti-VEGF treatment. In fact, in our series intra-retinal and sub-retinal fluid was scarce and clearly detectable in only a few cases.

To the best of our knowledge, there are no published studies describing outer retinal characteristics associated with active myopic CNV and their changes over time in eyes showing morphological (FA leakage) and functional (BCVA) improvement after anti-VEGF treatment. Our findings suggest that PM CNV may be visualized on SD-OCT as a hyper-reflective lesion with fuzzy borders, and a more highly reflective core above the RPE. After treatment, the fuzzy borders disappear and the highly reflective core may regress, resulting, in some cases, in RPE thickening. We hypothesize that the hazy appearance of normal retinal reflectivity may be caused by the outer retinal swelling associated with active neovascular proliferation. This seems to be confirmed by the disappearance of the fuzzy hyper-reflectivity, and by the recovery of normal outer retinal reflectivity after treatment. RPE thickening, which appears after antiVEGF treatment, may be due to the scarring inactive neovascular membrane and to reactive RPE hyperplasia.

SD-OCT allows visualization of what is supposed to be the IS/OS junction, or the ellipsoid portion of IS.7 Several authors have reported that IS/OS status correlates with visual acuity in various retinal diseases. ${ }^{19-22}$ Recently, Sayanagi et $a l^{23}$ showed that the IS/OS status appears to be a good indicator of visual outcome after anti-VEGF therapy in patients with wet AMD.

In our study, an 'absent or altered' IS/OS junction was associated with CNV activity. Despite the good sensitivity and specificity for CNV activity, we do not believe that an 'absent or altered' IS/OS junction might predict the treatment outcome in PM CNV, given that in the current series it was absent in most patients at baseline (19 out of 22 eyes).

Taken together, our findings suggest that in patients affected by myopic CNV, who are undergoing anti-VEGF treatment on PRN basis, one should look for the presence of a hyper-reflective lesion with fuzzy borders and an 'absent or altered' IS/OS junction as signs of CNV activity, rather than retinal thickness (unchanged after treatment), or the presence of sub-retinal/intra-retinal fluid (not associated with CNV activity)

Our study has several limitations, mainly due to the small number of studied eyes and the short follow-up. Further investigations are needed to improve our 
knowledge regarding the use of SD-OCT in the management of myopic CNV.

In conclusion, we showed that active myopic $\mathrm{CNV}$ are rarely associated with retinal fluid, and that outer retinal characteristics (ie, a hyper-reflective lesion with fuzzy borders, and an 'absent or altered' IS/OS junction) appear more meaningful than CRT in the evaluation of myopic CNV activity. These outer retinal characteristics on SD-OCT showed good overall sensitivity and specificity when compared with FA leakage (standard reference), and could be considered an alternative diagnostic tool to FA for myopic CNV monitoring.

\section{Summary}

\section{What was known before}

- OCT useful for CNV complicating AMD but not for CNV complicating PM.

- Fluorescein angiography gold standard for CNV in PM.

What this study adds

- No evidence of utility for central retinal thickness in PM-related CNV.

- No evidence of utility for intra- or sub-retinal fluid in PM-related CNV.

- Typical SD-OCT morphological myopic CNV appearance.

\section{Conflict of interest}

FB is an advisory board member for Alcon, Allergan, Bausch \& Lomb, Bayer, Novartis Pharma AG, Pfizer, and Thea. The other authors declare no conflict of interest.

\section{Acknowledgements}

We wish to thank Michael John of the Vita-Salute San Raffaele University for the English language editing of this manuscript.

\section{References}

1 Hampton GR, Kohen D, Bird AC. Visual prognosis of disciform degeneration in myopia. Ophthalmology 1983; 90(8): 923-926.

2 Avila MP, Weiter JJ, Jalkh AE, Trempe CL, Pruett RC, Schepens CL. Natural history of choroidal neovascularization in degenerative myopia. Ophthalmology 1984; 91(12): 1573-1581.

3 Yoshida T, Ohno-Matsui K, Yasuzumi K, Kojima A, Shimada N, Futagami $S$ et al. Myopic choroidal neovascularization: a 10-year follow-up. Ophthalmology 2003; 110(7): 1297-1305.

4 Blinder KJ, Blumenkranz MS, Bressler NM, Bressler SB, Donato G, Lewis $\mathrm{H}$ et al. Verteporfin therapy of subfoveal choroidal neovascularization in pathologic myopia: 2-year results of a randomized clinical trial-VIP report no. 3 . Ophthalmology 2003; 110(4): 667-673.
5 Cohen SY. Anti-VEGF drugs as the 2009 first-line therapy for choroidal neovascularization in pathologic myopia. Retina 2009; 29(8): 1062-1066.

6 Holz FG, Amoaku W, Donate J, Guymer RH, Kellner U, Schlingemann RO et al. Safety and efficacy of a flexible dosing regimen of ranibizumab in neovascular age-related macular degeneration: the SUSTAIN study. Ophthalmology 2011; 118(4): 663-671.

7 Spaide RF, Curcio CA. Anatomical correlates to the bands seen in the outer retina by optical coherence tomography: literature review and model. Retina 2011; 31(8): 1609-1619.

8 Martin DF, Maguire MG, Ying GS, Grunwald JE, Fine SL, Jaffe GJ. Ranibizumab and bevacizumab for neovascular age-related macular degeneration. N Engl J Med 2011; 364(20): 1897-1908.

9 Rosenfeld PJ, Brown DM, Heier JS, Boyer DS, Kaiser PK, Chung CY et al. Ranibizumab for neovascular age-related macular degeneration. $N$ Engl J Med 2006; 355(14): 1419-1431.

10 Brown DM, Kaiser PK, Michels M, Soubrane G, Heier JS, Kim RY et al. Ranibizumab vs verteporfin for neovascular age-related macular degeneration. N Engl J Med 2006; 355(14): 1432-1444.

11 Fung AE, Lalwani GA, Rosenfeld PJ, Dubovy SR, Michels S, Feuer WJ et al. An optical coherence tomography-guided, variable dosing regimen with intravitreal ranibizumab (Lucentis) for neovascular age-related macular degeneration. Am J Ophthalmol 2007; 143(4): 566-583.

12 Chan WM, Lai TY, Liu DT, Lam DS. Intravitreal bevacizumab (Avastin) for myopic choroidal neovascularization: six-month results of a prospective pilot study. Ophthalmology 2007; 114(12): 2190-2196.

13 Konstantinidis L, Mantel I, Pournaras JA, Zografos L, Ambresin A. Intravitreal ranibizumab (Lucentis) for the treatment of myopic choroidal neovascularization. Graefes Arch Clin Exp Ophthalmol 2009; 247(3): 311-318.

14 Silva RM, Ruiz-Moreno JM, Rosa P, Carneiro A, Nascimento J, Rito LF et al. Intravitreal ranibizumab for myopic choroidal neovascularization: 12-month results. Retina 2010; 30(3): 407-412.

15 Mones JM, Amselem L, Serrano A, Garcia M, Hijano M. Intravitreal ranibizumab for choroidal neovascularization secondary to pathologic myopia: 12-month results. Eye 2009; 23(6): 1275-1280; quiz 1281.

16 Gharbiya M, Allievi F, Mazzeo L, Gabrieli CB. Intravitreal bevacizumab treatment for choroidal neovascularization in pathologic myopia: 12-month results. Am J Ophthalmol 2009; 147(1): 84-93.e1.

17 Lalloum F, Souied EH, Bastuji-Garin S, Puche N, Querques G, Glacet-Bernard A et al. Intravitreal ranibizumab for choroidal neovascularization complicating pathologic myopia. Retina 2010; 30(3): 399-406.

18 Keane PA, Liakopoulos S, Chang KT, Heussen FM, Ongchin SC, Walsh AC et al. Comparison of the optical coherence tomographic features of choroidal neovascular membranes in pathological myopia vs age-related macular degeneration, using quantitative subanalysis. $\mathrm{Br} \mathrm{J}$ Ophthalmol 2008; 92(8): 1081-1085.

19 Witkin AJ, Ko TH, Fujimoto JG, Chan A, Drexler W, Schuman JS et al. Ultra-high resolution optical coherence tomography assessment of photoreceptors in retinitis pigmentosa and related diseases. Am J Ophthalmol 2006; 142(6): 945-952. 
20 Piccolino FC, de la Longrais RR, Ravera G, Eandi CM, Ventre L, Abdollahi A et al. The foveal photoreceptor layer and visual acuity loss in central serous chorioretinopathy. Am J Ophthalmol 2005; 139(1): 87-99.

21 Villate N, Lee JE, Venkatraman A, Smiddy WE. Photoreceptor layer features in eyes with closed macular holes: optical coherence tomography findings and correlation with visual outcomes. Am J Ophthalmol 2005; 139(2): $280-289$.
22 Inoue M, Watanabe Y, Arakawa A, Sato S, Kobayashi S, Kadonosono K. Spectral-domain optical coherence tomography images of inner/outer segment junctions and macular hole surgery outcomes. Graefes Arch Clin Exp Ophthalmol 2009; 247(3): 325-330.

23 Sayanagi K, Sharma S, Kaiser PK. Photoreceptor status after antivascular endothelial growth factor therapy in exudative age-related macular degeneration. Br J Ophthalmol 2009; 93(5): 622-626. 